\title{
Case-based reasoning approach for utilisation of past remarks as advice for collaborative learning
}

\section{Tomoko Kojiri and Shuto Ohata}

Faculty of Engineering Science,

Kansai University,

3-3-35 Yamate-cho,

Suita, Osaka, 564-8680, Japan

E-mail: kojiri@kansai-u.ac.jp

E-mail: k465636@kansai-u.ac.jp

*Corresponding author

\section{Yuki Hayashi}

Faculty of Science and Technology,

Seikei University,

3-3-1 Kichijoji-Kitamachi,

Musashino-shi, Tokyo 180-8633, Japan

E-mail: hayashi@st.seikei.ac.jp

\begin{abstract}
The collaborative learning remarks that were triggers of the past active discussion, which is called discussion promotion remark, can also be effective in the current discussion situation whose speech patterns are similar. The objective of this research is to construct the advising system for the collaborative learning which utilises discussion promotion remarks of the past collaborative learning as advice to solve current inappropriate situation. Discussion consists of various characteristics, so it is difficult to define appropriate speech pattern for each type of discussion promotion remark. This research introduces case-based reasoning approach to extract past discussion promotion remark which can solve current inappropriate situation. This paper describes two of the steps to accomplish the case-based reasoning system. First, several parameters that characterise the discussion situation are introduced and attached to past discussion promotion remarks. Second, discussion promotion remark database is constructed as a decision tree based on the attached parameters.
\end{abstract}

Keywords: discussion support; case-based reasoning; discussion promotion remark; collaborative learning.

Reference to this paper should be made as follows: Kojiri, T., Ohata, S. and Hayashi, Y. (2014) 'Case-based reasoning approach for utilisation of past remarks as advice for collaborative learning', Int. J. Knowledge and Web Intelligence, Vol. 5, No. 1, pp.36-48.

Biographical notes: Tomoko Kojiri received his $\mathrm{BE}, \mathrm{ME}$ and $\mathrm{PhD}$ degrees from Nagoya University, Japan in 1998, 2000, and 2003, respectively. In 2002, she was a Research Fellow of the Japan Society for the Promotion of Science. From 2003 to 2007, she was a Research Associate with Nagoya University. From 2007 to 2011, she was an Assistant Professor with Nagoya University. From 2011, she has been an Associate Professor with the Faculty of 


\begin{abstract}
Engineering Science, Kansai University, Japan. Her current research interests include computer-supported collaborative learning, intelligent tutoring system, meta-learning support, and human computer interface. She has received several awards, including Outstanding Paper Award of ICCE/ICCAI 2000, Best Paper Award of KES 2005, and Outstanding Poster Presentation Award of ICCE 2007. She is a member of APSCE, IEICE, IPSJ, JSAI, JSET, JSiSE and KES International.
\end{abstract}

Shuto Ohata received his BE degree from Kansai University, Japan in 2013. He is now with Fujitsu FSAS Inc.

Yuki Hayashi received his BE, ME, and $\mathrm{PhD}$ from Nagoya University, Japan, in 2007, 2009, and 2012, respectively. Currently, he is an Assistant Professor at Seikei University, Japan. His research interests include computer-supported collaborative learning and computer human interaction. He is a member of IPSJ, JSAI, HIS and JSiSE.

This paper is a revised and expanded version of a paper entitled 'Utilization of past remarks of similar speech pattern as advice for collaborative learning' presented at the KES/IIMSS2013 Conference, Sesimbra, June 2013.

\title{
1 Introduction
}

Active discussion is the key to the successful collaborative learning. Learners need to participate into the discussion actively and exchange opinions in the limited discussion time. However, especially in the collaborative learning in the distributed environment, to keep active discussion is difficult since there is in many cases no teacher or mentor who leads the discussion (Dillenbourg, 1999; Koschmann, 1996). Remarks that were triggers of the past active discussion, which is called discussion promotion remark, can also be effective in the current discussion if speech patterns of their situations were similar. Thus, the objective of this research is to construct the system which utilises discussion promotion remarks of the past collaborative learning as advice to solve the current inappropriate situation.

Various researches aim at utilising collaborative learning history. Several researches tried to extract meaningful learning contents in order to apply them for the future learning. Most of them are annotating systems in which learners can actively attach annotations to the effective contents (Kakehi and Lee, 2006; Kunimune et al., 2010). Effective learning contents may be gathered easily using these systems. Since effective contents are different among learners, annotated contents are not always applicable. Watanabe et al. (2010) proposed the mechanism which extracts remarks that contain knowledge for solving programming exercise. The extracted remarks could give hints for solving the exercise. However, these researches were not able to control inappropriate discussion situation, for example, the existence of members who do not actively participating into the discussion.

In order to control the discussion, several researches defined inappropriate discussion situation and prepared advices for each situation statically (Suh et al., 2006; Ayala and Yano, 1998). For example, Okamoto and Inaba (1996) defined four inappropriate situations: answer is not derived, the numbers of remarks are not equal for all members, 
focuses of remarks are not corresponded, and some questions are not solved. Then, they prepared advices for each situation. The advices may solve the defined situation. However, since situation consists of various factors, advices are sometimes not appropriate for the defined situation. In addition, they do not cope with inappropriate situation that are not defined.

This research utilises discussion promotion remarks of the past collaborative learning to solve inappropriate situation of the current discussion. Discussion consists of various characteristics, so it is difficult to define appropriate speech pattern for each type of discussion promotion remark. This research introduces case-based reasoning approach to extract past discussion promotion remark that corresponds to the speech pattern for the current inappropriate situation.

Several researches introduced case-based reasoning to derive the answer of the current problem, such as failure diagnosis of electric circuit or disease diagnosis (Bradbum and Zeleznikow, 1994; Hammond, 1986; Kolodner, 1992). In these targets, characteristics of problems are easy to describe. However, discussion consists of various factors, so it is difficult to define characteristics that represent the speech pattern. In this paper, firstly, characteristics of representing the speech pattern are proposed. The database structure of the discussion promotion remarks is introduced. Then, as an application, database of the discussion promotion remarks for the consensus game is developed and its quality is evaluated.

\section{Approach}

\subsection{Discussion promotion remarks}

Discussion promotion remark is the remark which changes the discussion situation from inappropriate one to appropriate one as shown in Figure 1. Currently, we focus on the discussion for solving the problem that has plural answers. The objective of the discussion is to derive one group answer by persuading their opinions with each other. In such situation, inappropriate situation and appropriate situation are difficult to describe. We define the inappropriate situation is the situation that many learners thought inappropriate, e. g. discussion is not activate or it is difficult to get agreement in group. Appropriate situation is the situation that solves the inappropriateness of its former situation. Discussion promotion remarks are those, learners thought, that solve the inappropriateness of the former situation.

Figure 1 Discussion promotion remark (see online version for colours)

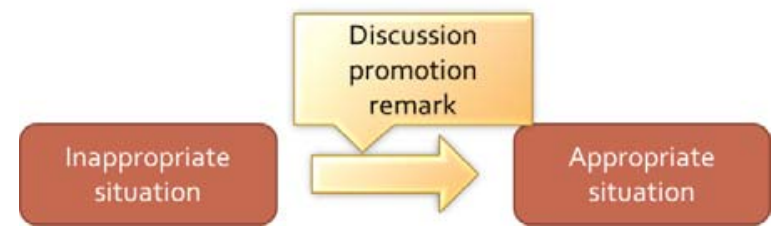

Types of discussion promotion remarks are characterised by the types of inappropriate discussion situations that they solved. Discussion situation can be grasped by the speech pattern of remarks. Therefore, discussion promotion remarks are regarded as the solution 
of the speech patterns of its former remarks. In order to characterise discussion promotion remarks, they are stored with characteristics of its former remarks that represent the speech pattern. Then, the objective of making remarks is to solve the inappropriateness of the situation, which is discriminated by its former remarks.

\subsection{Framework of discussion promotion remarks}

Appropriate discussion promotion remarks of the past collaborative learning are those whose speech patterns of the inappropriate situations are similar to the current situation. In order to detect discussion promotion remarks that have similar speech pattern, case-based reasoning approach is introduced. In this approach, past discussion promotion remarks are organised in the case-base based on the speech patterns of the inappropriate situation. Then, past discussion promotion remarks whose speech patterns are similar to the current situation are selected as triggers of solving current inappropriate situation.

Figure 2 shows the framework of the system. Solid lines represent control flow and dashed lines indicate data references. System holds the database of discussion promotion remarks of the past collaborative learning. Discussion promotion remarks are selected by learners in the past collaborative learning and are classified with their types. Each discussion promotion remark is tagged with the parameters that represent speech pattern of its former remarks. The parameters are called characteristic parameters.

Figure 2 Framework of system (see online version for colours)

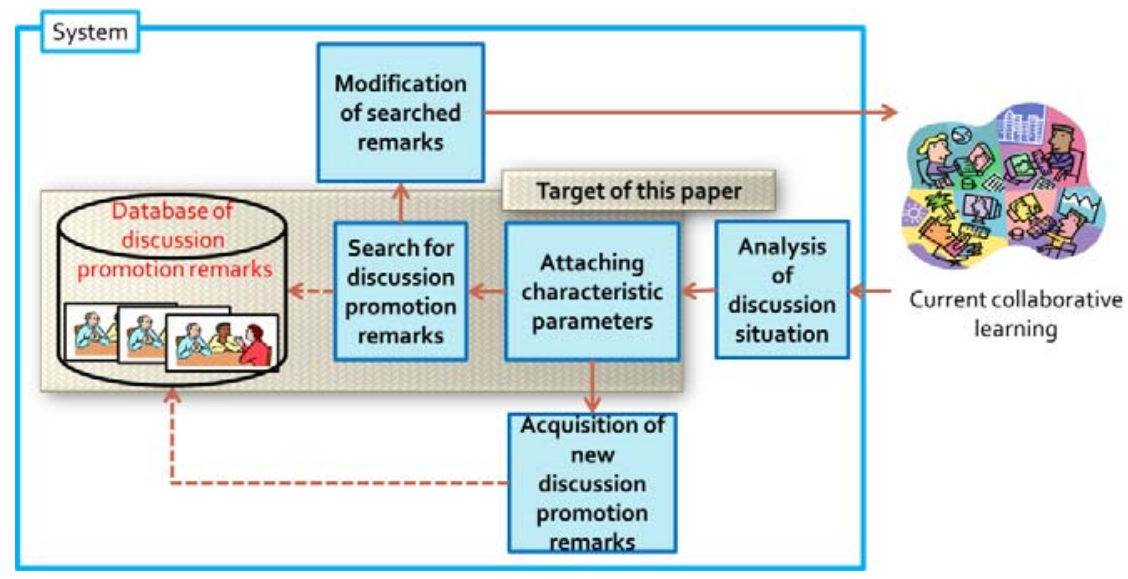

When inappropriate discussion situation is detected by the analysis module, characteristic parameters for the current remarks are attached. By inputting the characteristic parameters, a category of the past discussion promotion remarks is selected from the database whose characteristic parameters are similar. Past discussion promotion remarks contain some words that are specific to the members group such as the name of the participant, or the topic. So, the system modifies the searched remarks so as to fit for the current collaborative learning. For example, if the remark " $<$ Person name $>$, give me your opinion." is selected, the system exchanges $<$ Person name $>$ into the name of the current member, e.g., $M r$. $A$. In addition, if the system detects new discussion promotion remarks when analyzing the situation, it acquires the remarks, assigns them to the category of the discussion promotion remarks, and stores them into the database. 
'Eliza' is a famous interaction system which creates human-like responses based on simple mechanisms, such as the string substitution and keyword-based responding method (Weizenbaum, 1966). This system only gives supportive responses, since its objective is to make communicating partner keep talking about themselves. However, our system can give remarks that can solve the inappropriate situation.

This paper focuses on defining characteristic parameters and constructs the database of the discussion promotion remarks. In addition, search module is developed which selects discussion promotion remark by input characteristic parameter.

\section{Database for discussion promotion remarks}

\subsection{Characteristic parameters}

Discussion promotion remarks of the past collaborative learning are characterised by the speech pattern of the remarks that were input before the discussion promotion remarks. There are various factors that characterise the discussion situation. In the research field of computer supported collaborative learning (CSCL), various researches proposed various factors to evaluate the learning (Okamoto and Inaba, 1996; Hayashi et al., 2013; Conklin and Begeman, 1988; Niki et al., 2011). In the final system, characteristic parameters are attached by the system. So, they should be simple enough to be attached by the system automatically. Table 1 shows the defined characteristic parameters.

Table 1 Characteristic parameters

\begin{tabular}{|c|c|c|}
\hline $\begin{array}{l}\text { Types of characteristic } \\
\text { parameter }\end{array}$ & Meaning & Values \\
\hline Discussion phase & $\begin{array}{l}\text { Typical types of discussion which can be seen } \\
\text { along time sequence. In the sharing phase, } \\
\text { members share own ideas. In diffusion phase, } \\
\text { various opinions are discussed based on members' } \\
\text { ideas. In the conclusion phase, group answer is } \\
\text { determined. }\end{array}$ & $\begin{array}{l}\text { Sharing phase } \\
\text { Diffusion phase } \\
\text { Conclusion phase }\end{array}$ \\
\hline Frequency of remarks & $\begin{array}{l}\text { Duration from last remark and discussion } \\
\text { promotion remark. Duration between two remarks } \\
\text { represents whether the discussion is active or not. }\end{array}$ & $\begin{array}{l}\text { Short } \\
\text { Normal } \\
\text { Long }\end{array}$ \\
\hline $\begin{array}{l}\text { Balance of remarks for } \\
\text { each member }\end{array}$ & $\begin{array}{l}\text { Balance of the number of remarks for each } \\
\text { member. If only a few members input remarks, } \\
\text { the discussion is unbalanced. }\end{array}$ & $\begin{array}{c}\text { Unbalanced } \\
\text { Balanced }\end{array}$ \\
\hline Remark types & $\begin{array}{l}\text { Sequence of remark types, which represents } \\
\text { discussion situation from semantically. }\end{array}$ & $\begin{array}{l}\text { Number of remarks } \\
\text { for each remark } \\
\text { type }\end{array}$ \\
\hline
\end{tabular}

\subsection{Database structure}

In order to organise categories of the discussion promotion remarks, the decision tree is introduced. A decision tree is a decision support tool that uses a tree-like graph or model of decisions and their possible consequences and is used for several researches that apply machine learning techniques (Magerman, 1995; Safavian and Landgrebe, 1991; Friedl 
and Brodley, 1997). Figure 3 is the imagination of the decision tree in our system. Categories are set as leaf nodes. Root node and intermediate nodes correspond to the characteristic parameters that identify the categories. Conditions are attached to links that are used to select categories based on the characteristic parameters.

Figure 3 Imagination of decision tree (see online version for colours)

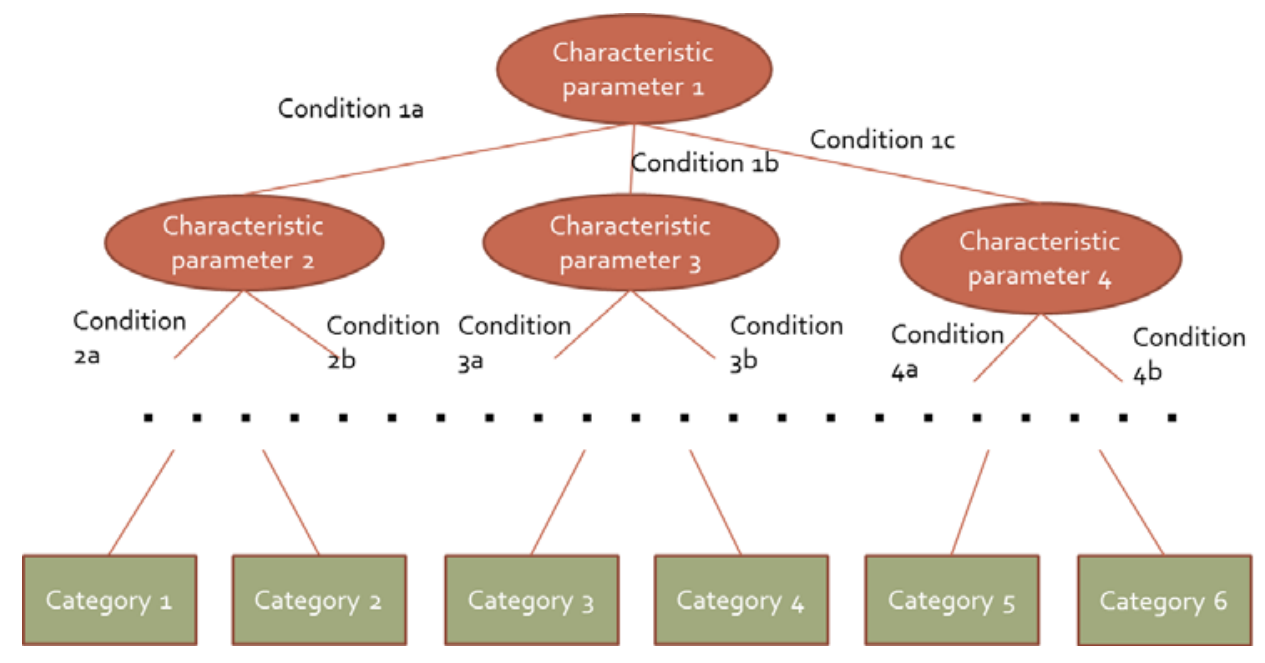

\section{Implementation example: collaborative learning support system for consensus game}

\subsection{Database structure}

We have developed the advising system using the past discussion promotion remarks for the discussion of the consensus game. Consensus game is a kind of a theme for which members in a group discuss to derive one group answer from various candidates. Members are required to have abilities of explaining own ideas, listening to other ideas, and organising various different ideas, so the theme is applied to various researches in computer-supported collaborative learning or computer-supported cooperative work (Conklin and Begeman, 1988; Bochman, 2012). In our research, members were asked to select three items from lists with order for surviving from jungle. Candidates are flash light, map, pistol, parachute, vodka, salt, coat, mirror, compass, and book of mushroom. Main focus of the discussion is to get agreement of others by exchanging the opinions.

In order to collect discussion promotion remarks for the initial system, five groups of four university students were asked to discuss about the topic. They were asked to discuss 30 minutes after deciding their opinions for seven minutes. In order to gather discussion promotion remarks, we have constructed a chat system. Figure 4 is the interface of the chat system. It is a semantic chat system (Yuizono et al., 2005) in which members input remarks with their types selected from the list. Currently, we have prepared ten remark types; proposition, agreement, disagreement, question, answer, complement remark, leading remark, conclusion, and others. Remarks were recorded with their time stamps. 
Figure 4 Interface of chat system (see online version for colours)

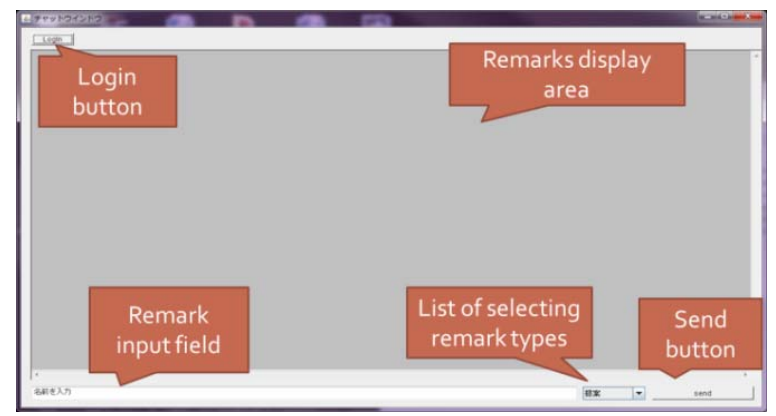

After the discussion, there were asked to point out remarks that promoted the discussion. Remarks that were pointed out by more than two members were selected as discussion promotion remarks. As a result, 55 remarks were selected. Based on their contents, they were classified into 14 categories as shown in Table 2.

Table 2 Categories of discussion promotion remarks

\begin{tabular}{lcc}
\hline Category & Example of remarks & $\begin{array}{c}\text { Number of } \\
\text { selected remarks }\end{array}$ \\
\hline Requirement of opinion & "Give me your opinion." & 7 \\
Requirement of reason & "Why do you select X?" & 7 \\
Ask for agreement & "Do we all agree with X?" & 7 \\
Confirmation of answer & "Do you mean X?" & 7 \\
Time management & "We have only 10 minutes." & 5 \\
Proposition of discussion policy & "Let's list up impossible items." & 5 \\
Question for other remarks & "Why did you select X?" & 4 \\
Promotion of ordering & "Let's order selected items." & 3 \\
Proposition of items & "I think X is the most important items." & 3 \\
Elimination of items & "X is not important if Y is selected." & 2 \\
Proposing alternatives & "Which do you select, X or Y?" & 2 \\
Confirmation of current situation & "One more candidate to go." & 1 \\
Focusing discussion topic & "What do you mean by X?" & 1 \\
Questioning for items & "Let's think of how to use X?" & 1 \\
\hline
\end{tabular}

\subsection{Structure of decision tree for discussion promotion remarks}

Characteristic parameters were added to selected discussion promotion remarks. In this case, characteristic parameters were added based on the five remarks that were input before the discussion promotion remarks. Criteria of attaching characteristic parameters were shown in Table 3. 
Table 3 Criteria of attaching characteristic parameters

\begin{tabular}{lcc}
\hline $\begin{array}{l}\text { Types of characteristic } \\
\text { parameter }\end{array}$ & Values & Criteria \\
\hline Discussion phase & Sharing phase & 0 to 3 minutes from start \\
& Diffusion phase & 3 to 23 minutes from start \\
& Conclusion phase & 23 to 30 minutes from start \\
Frequency of remarks & Short & 0 to 9 seconds \\
& Normal & 10 to 29 seconds \\
Balance of remarks for each & Long & More than 30 seconds \\
member & Unbalanced & Existence of member who input \\
& Balanced & more than three remarks \\
Remark types & & All members input three remarks \\
& Number of remarks for each & Remark types selected by \\
& remark type & members when inputting remarks \\
\hline
\end{tabular}

Here, we explain the example of attaching characteristic parameters using discussion example shown in Table 4. This discussion example assumes discussion of 30 minutes and the number of its participants is four $(A, B, C$, and $D)$. Let is assume last remark, i.e., leading remark, is selected as a discussion promotion remark. The characteristic parameter of discussion phase is attached as sharing phase, since discussion promotion remark was input before three minutes from the start of the discussion. The characteristic parameter of frequency of remarks is long, because duration from its former remark is 39 seconds. The characteristic parameter of balance of remarks is balanced, because all members input more than one remarks in five remarks before the discussion promotion remark. The characteristic of remark types was ((Question, 1), (Answer, 4)).

Table 4 Discussion example

\begin{tabular}{|c|c|c|c|}
\hline Time & Speaker & Type of remark & Contents \\
\hline $1: 31$ & $\mathrm{C}$ & Answer & For me, 1 st is $\circ \circ, 2 \mathrm{nd}$ is $\triangle \triangle, 3 \mathrm{rd}$ is $\square \square$ \\
\hline $1: 32$ & A & Answer & For me, 1 st is $\triangle \triangle, 2$ nd is $\bullet \bullet, 3$ rd is $\circ \circ$ \\
\hline $1: 55$ & $\mathrm{C}$ & Question & What do you think? \\
\hline $2: 10$ & B & Answer & 1 st is $\triangle \triangle, 2$ nd is $\bullet \bullet, 3 \mathrm{rd}$ is $\circ \circ$ \\
\hline $2: 13$ & $\mathrm{D}$ & Answer & 1 st is $\square \square, 2$ nd is $\bullet \bullet, 3$ rd is $\circ \circ$ \\
\hline $2: 52$ & $\mathrm{C}$ & Leading remark & Let's give us the reasons! \\
\hline
\end{tabular}

Data mining tool, (Weka 3), is used to create decision tree for the categories of selected discussion promotion remarks. J48 algorithm is applied to construct the decision tree. Figure 5 is the structure of created decision tree. It consists of 11 intermediate nodes that indicate the characteristic parameters, and 15 leaf nodes that correspond to categories. 
Figure 5 Created decision tree (see online version for colours)

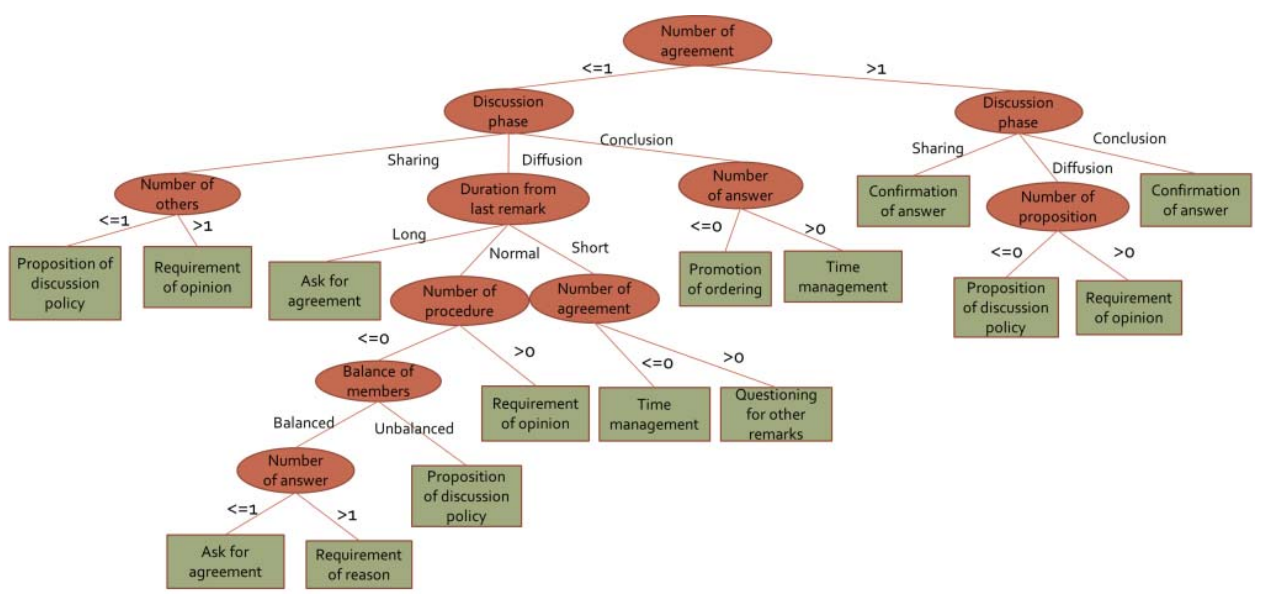

Figure 6 Sheet of discussion history (see online version for colours)

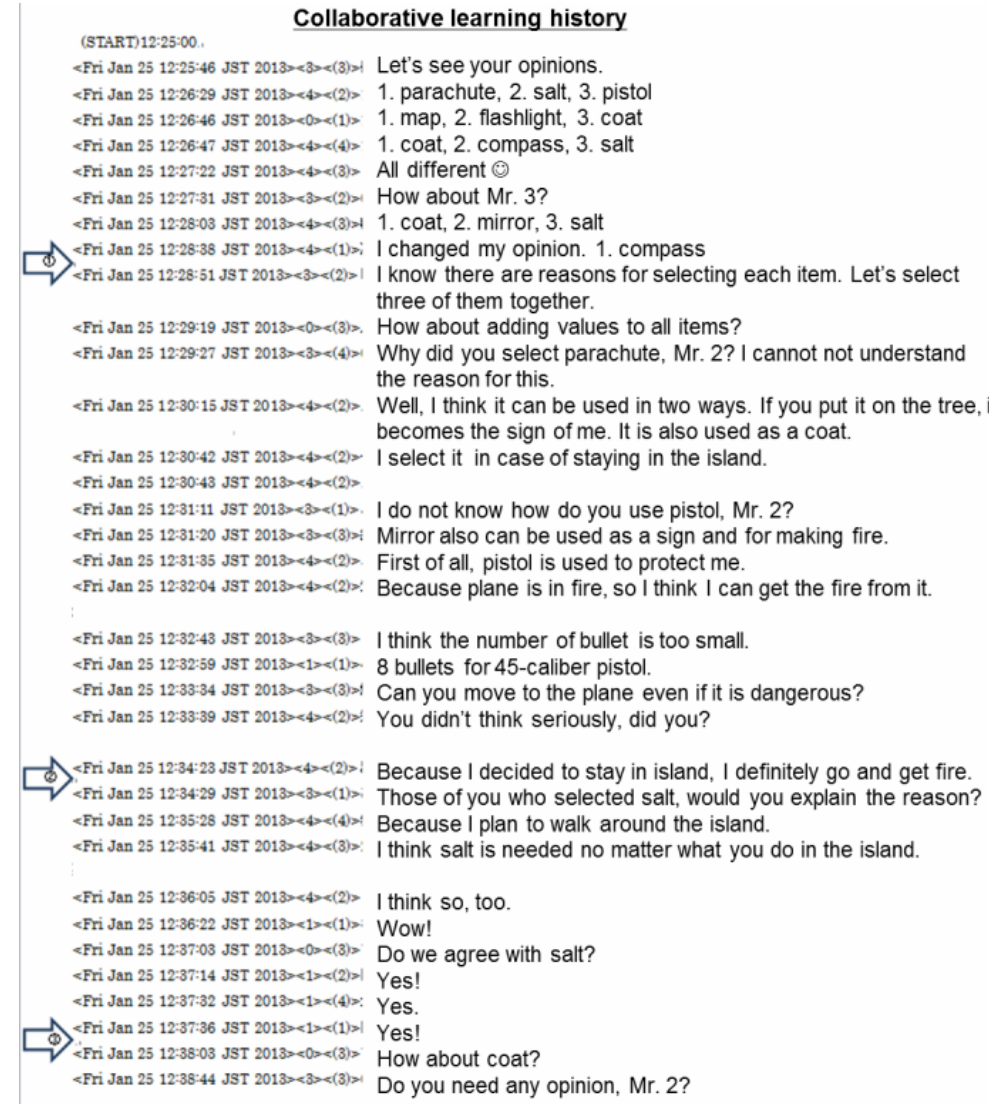




\subsection{Evaluation of created decision tree}

We have evaluated the effectiveness of created decision tree. System for selecting categories from input characteristic parameters were developed. Output categories for input characteristic parameters were evaluated if they could solve the situation corresponded to the characteristic parameters. In order to collect inappropriate situations, four university students were asked to discuss the same consensus game. After the discussion, they were asked to point out remarks from the discussion history at which they thought discussion situation was inappropriate, which is called inappropriate points. Figure 6 is a sheet of the discussion history which they used to point out inappropriate points and arrows are indicated inappropriate point. Five inappropriate points were selected.

Figure 7 is the interface of the system. This system is used for searching category of discussion promotion remarks for the current situation represented by input parameters. Characteristic parameters of the current situation were input at the upper half of the interface. According to the input parameters, the system traverses decision tree from the root node to the leaf node. Firstly, the system follows link from the root node that satisfies the current parameters. Again, the system follows the link from the current node that also satisfies the current parameters. System repeats this process until reaching to the leaf node which corresponds to categories of discussion promotion remarks. Then, the searched category and discussion promotion remarks that belong to the category are shown at the lower half of the interface.

Figure 7 Interface for searching category from decision tree (see online version for colours)

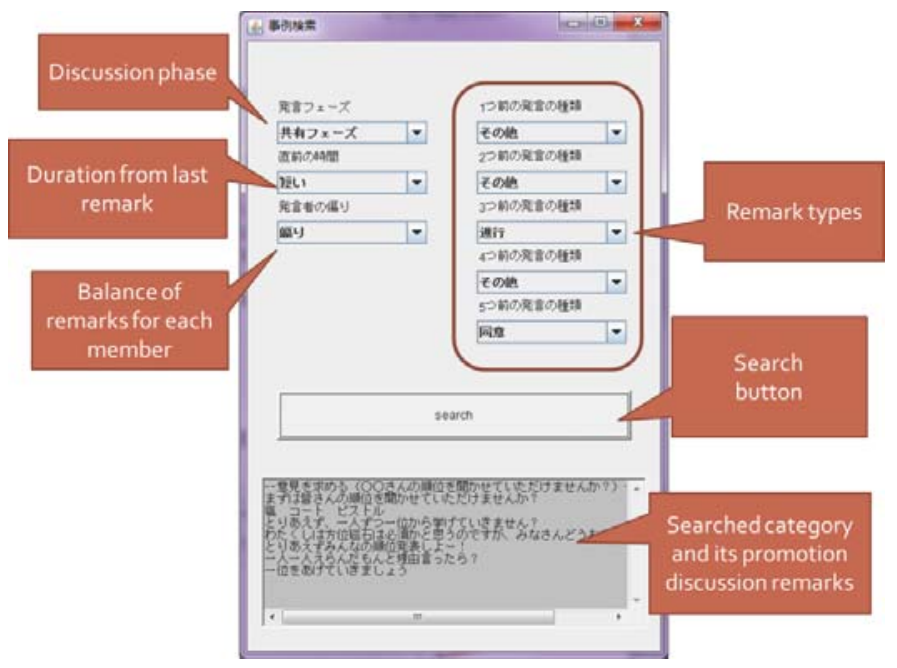

Members were asked to answer the questionnaire if promotion discussion remarks of searched category could solve the input situation. They were asked to select one from five values. Figure 8 shows the questionnaire sheet. Table 5 shows the result. Inappropriate situations of No. 1 and 3 got good answers, while the system could not derive appropriate categories for other situations. Inappropriate situation of No. 1 was 
happened in the sharing phase, and that of No. 3 belongs to the conclusion phase. In such phases, since there were not so many opinions, typical advices may be restricted so that the system could derive them successfully. However, during the diffusion phase, various remarks may be derived, so it is difficult to determine typical advices. Moreover, since our current system does not have enough remarks for the categories, the appropriate categories were not detected. We need to collect more discussion promotion remarks for each category.

Figure 8 Questionnaire sheet (see online version for colours)

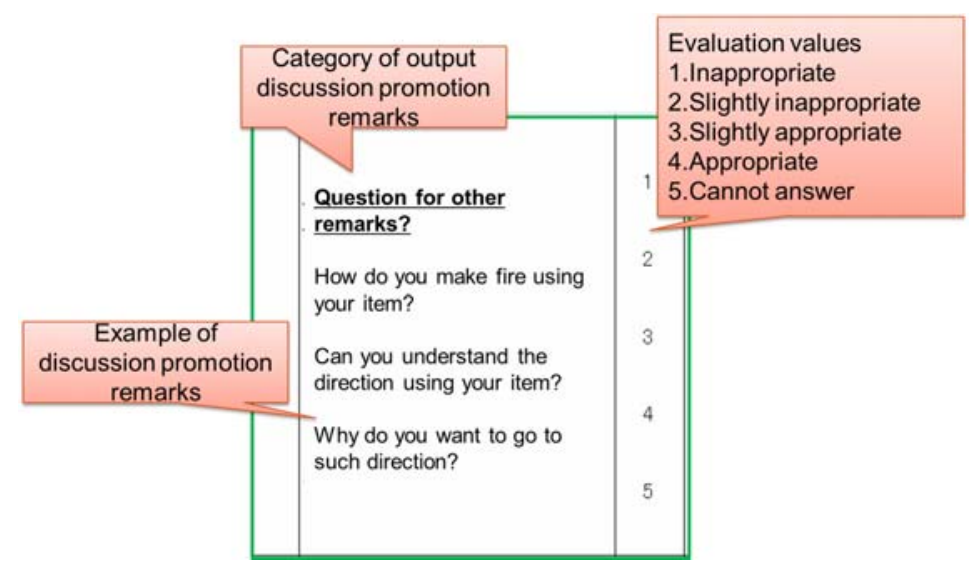

Note: Contents are translated into English.

Table 5 Result of questionnaire

\begin{tabular}{clccccc}
\hline No. & $\begin{array}{c}\text { Detected } \\
\text { category }\end{array}$ & Inappropriate & $\begin{array}{c}\text { Slightly } \\
\text { inappropriate }\end{array}$ & $\begin{array}{c}\text { Slightly } \\
\text { appropriate }\end{array}$ & Appropriate & $\begin{array}{c}\text { Can't } \\
\text { answer }\end{array}$ \\
\hline 1 & $\begin{array}{l}\text { Proposition of } \\
\text { discussion } \\
\text { policy }\end{array}$ & 0 & 0 & 0 & 4 & 0 \\
2 & $\begin{array}{l}\text { Questioning for } \\
\text { other remarks } \\
\text { Requirement of } \\
\text { opinion }\end{array}$ & 0 & 2 & 1 & 0 & 1 \\
4 & 1 & 0 & 2 & 0 & 0 \\
\hline $\begin{array}{l}\text { Time } \\
\text { management }\end{array}$ & $\begin{array}{l}\text { Time } \\
\text { management }\end{array}$ & 0 & 0 & 0 & 4 & 0 \\
\hline
\end{tabular}

In order to evaluate the characteristic parameter, especially remark types, members were also asked to answer questionnaire; "Do you think prepared remark types are appropriate?". Members were asked to select one from five answers, such as inappropriate, slightly inappropriate, slightly appropriate, appropriate and others. As a result, two members selected 'appropriate' and other two members did 'slightly appropriate'. It means that members were able to select remark types easily when inputting remarks, so prepared remarks were appropriate. 


\section{Conclusions}

In this paper we proposed the advising mechanism using past discussion promotion remarks based on the case-based reasoning framework. In addition, we have developed the system for the discussion topic of the consensus game. The system selected categories of discussion promotion remarks that correspond to the input characteristic parameters. Although, the numbers of stored remarks for each category were small, detected categories were appropriate for the discussion situations in the sharing phase and conclusion phase. We need further evaluation with more remarks. Also, we need to evaluate the effectiveness of the system by comparing human facilitators.

Currently, characteristic parameters were attached manually to the situation. Also, inappropriate situations were not able to be detected by the system. Some types of inappropriate situations can be defined by the sequence of the speech patterns. So, in our future work, we need to develop the system which selects inappropriate situations based on the speech patterns and automatically searches for the categories of the discussion promotion remarks based on their speech patterns.

Current our system focuses on the discussion topic of the consensus game. However, the consensus game is not often discussed in our daily life. In order to make this system usable, we need to find practical discussion field for the system.

\section{References}

Ayala, G. and Yano, Y. (1998) 'A collaborative learning environment based on intelligent agents', Expert Systems with Applications, Vol. 14, Nos. 1-2, pp.129-137.

Bochman, O. (2012) 'The consensus game: modeling peer decision protocols', WikiSym '12 Proceedings of the Eighth Annual International Symposium on Wikis and Open Collaboration, Article No. 37.

Bradbum, C. and Zeleznikow, J. (1994) 'The application of case-based reasoning to the tasks of health care planning', Proc. of EWCBR-93, pp.365-378.

Conklin, J. and Begeman, M.L. (1988) 'gIBIS: a hypertext tool for exploratory policy discussion', CSCW '88 Proceedings of the 1988 ACM Conference on Computer-Supported Cooperative Work, pp.140-152.

Dillenbourg, P. (Ed.) (1999) Collaborative Learning - Cognitive and Computational Approaches, Elsevier Science Ltd., Oxford.

Friedl, M.A. and Brodley, C.E. (1997) 'Decision tree classification of land cover from re-motely sensed data', Remote Sensing of Environment, Vol. 61, No. 3, pp.399-409.

Hammond, K.J. (1986) 'CHEF: a model of case-based planning', Proc. of AAAI-86, pp.267-271.

Hayashi, Y., Ogawa, Y. and Nakano, Y.I. (2013) 'An experimental environment for analyzing collaborative learning interaction', Proc. of 15th International Conference on HumanComputer Interaction (HCII2013), Lecture Notes in Computer Science, Vol. 8018, pp.43-52.

Kakehi, M., Kojiri, T. and Watanabe, T. (2006) 'Time-based self-learning support using collaborative learning process', Proc. of ICCE 2006, pp.237-240, IOSPress.

Kolodner, J.L. (1992) 'An introduction to case-based reasoning', Artificial Intelligence Review, Vol. 6, No. 1, pp.3-34.

Koschmann, T. (Ed.) (1996) CSCL: Theory and Practice of an Emerging Paradigm, Lawrence Erlbaum Associates Publishers, Mahwah, New Jersey.

Kunimune, H., Takizawa, T. and Fuwa, Y. (2010) 'Implementation and evaluation of a method for realigning annotations in updated web-based materials', Intelligent Decision Technologies, Vol. 4, No. 4, pp.261-267. 
Magerman, D.M. (1995) 'Statistical decision-tree models for parsing', ACL '95 Proceedings of the 33rd Annual Meeting on Association for Computational Linguistics, pp.276-283.

Niki, K., Furuta, T., Akakura, T., Tomoto, T., Nishihori, Y. and Nagaoka, K. (2011) 'Method for evaluating participants' roles in online text discussion using network analysis', Proc. of ICCE 2011, pp.199-203.

Okamoto, T. and Inaba, A. (1996) 'The intelligent discussion supporting system under the distributed environment', Computer Aided Learning and Instruction in Science and Engineering, LNCS, Vol. 1108, pp.123-131.

Safavian, S.R. and Landgrebe, D. (1991) 'A survey of decision tree classifier methodology', IEEE Transactions on Systems, Man and Cybernetics, Vol. 21, No. 3, pp.660-674.

Suh, H.J. and Lee, S.W. (2006) 'Collaborative learning agent for promoting group interaction', ETRI Journal, Vol. 28, No. 4, pp.461-474.

Watanabe, Y., Kojiri, T. and Watanabe, T. (2010) 'Effective solution knowledge organization from discussion record', International Journal of Intelligent Decision Technologies, Vol. 4, No. 4, pp.241-251, IOS Press.

Weizenbaum, J. (1966) 'ELIZA - a computer program for the study of natural language communication between man and machine', Communications of the ACM, Vol. 9, No. 1, pp.36-45.

Weka 3, Data Mining with Open Source Machine Learning Software in Java [online] http://www.cs.waikato.ac.nz/ml/weka/ (accessed 2012).

Yuizono, T., Kayano, A., Shigenobu, T., Yoshino, T. and Munemori, J. (2005) 'Groupware for a new idea generation with the semantic chat conversation data', Knowledge-Based Intelligent Information and Engineering Systems, Lecture Notes in Computer Science, Vol. 3681, pp.1044-1050. 